\title{
Spectral and stochastic properties of the $f$-Laplacian, solutions of PDEs at infinity and geometric applications
}

\author{
G. Pacelli Bessa, Stefano Pigola and Alberto Setti
}

\begin{abstract}
The aim of this paper is to suggest a new perspective to study qualitative properties of solutions of semilinear elliptic partial differential equations defined outside a compact set. The relevant tools in this setting come from spectral theory and from a combination of stochastic properties of the differential operators in question. Possible links between spectral and stochastic properties are analyzed in detail.
\end{abstract}

\section{Introduction}

In this paper we will suggest a new viewpoint for studying qualitative properties of solutions of semilinear elliptic PDEs, especially when these are defined only outside a compact set. In order to enlarge the range of applicability of the techniques, we decide to place our treatment in the setting of weighted Riemannian manifolds and the corresponding drifted Laplacians.

The germ of the present investigation is contained in the very recent paper [20] which is devoted to a systematic treatment of the Feller property of a Riemannian manifold. In fact, using a suitable comparison theory, we will show how (weighted) manifolds which are both stochastically complete and Feller provide a natural framework where solutions of PDEs at infinity can be studied. The fact that transience and stochastic completeness of the underlying manifolds have PDEs counterparts is well understood. However, due to the nature of these stochastic properties, so far only global solutions have been considered. The introduction of the Feller property, in combination with stochastic completeness, will enable us to get important information even in the case of solutions at infinity.

Manifolds which are both stochastically complete and Feller belong to a fairly wide class containing complete Ricci solitons, complete manifolds with controlled

Mathematics Subject Classification (2010): Primary 58J05, 58J50; Secondary 60D05.

Keywords: Weighted Laplacians, Feller property, stochastic completeness, essential spectrum, gradient Ricci solitons. 
Ricci tensor and Cartan-Hadamard manifolds with at most quadratic exponential volume growth. The usefulness of the technique is visible in a wide range of geometric applications, from submanifold theory to the Yamabe problem. It is well known that the spectral theory of diffusion operators is affected by stochastic properties of the corresponding diffusion process. For instance, using capacitary arguments, it is readily seen that recurrence forces the bottom of the spectrum of a diffusion operator to be zero. The recent works [4] and [13] have emphasized an intriguing link between the stochastic completeness of certain specific manifolds and the essential spectrum of the operator. We will analyze, in some detail, possible relations between stochastic and spectral properties of weighted manifolds. On the route we will prove a generalized and abstract version of the discreteness of the spectrum of bounded minimal submanifolds recently obtained in [4]. The nature of the essential spectrum also suggests that it could fit in very well in the main topic of the paper. Indeed, by the decomposition principle, the bottom of the essential spectrum is sensitive only to the geometry at infinity of the underlying manifold and Barta's classical characterization leads naturally to solutions at infinity of PDEs.

Part of the present work was presented by the third named author at the workshop "Ricci solitons days" held in Pisa, April 4-8, 2011.

\section{Notation}

Throughout this paper, we shall use the symbol $M_{f}$ to denote the $m$-dimensional weighted manifold

$$
M_{f}=\left(M,\langle,\rangle, d \operatorname{vol}_{f}\right),
$$

where $(M,\langle\rangle$,$) is a Riemannian manifold, f: M \rightarrow \mathbb{R}$ is a selected smooth function on $M, d$ vol denotes the Riemannian measure of $(M,\langle\rangle$,$) and$

$$
d \operatorname{vol}_{f}=e^{-f} d \mathrm{vol}
$$

is the weighted measure. The $f$-Laplacian associated to the weighted manifold $M_{f}$ is the operator

$$
\Delta_{f} u=\operatorname{div}_{f}(\nabla u):=e^{f} \operatorname{div}\left(e^{-f} \nabla u\right),
$$

which is symmetric on $L^{2}\left(M, d \mathrm{vol}_{f}\right)$.

The Bakry-Emery Ricci curvature of the weighted manifold $M_{f}$ is the 2-tensor

$$
\operatorname{Ric}_{f}=\operatorname{Ric}+\operatorname{Hess}(f) .
$$

If

$$
\operatorname{Ric}_{f}=\lambda\langle,\rangle
$$

for some constant $\lambda \in \mathbb{R}$, then the weighted manifold $M_{f}$ is called a Ricci soliton. The Ricci soliton $M_{f}$ is said to be shrinking, steady, or expanding according to whether $\lambda>0, \lambda=0$, or $\lambda<0$, respectively. 


\section{Stochastic completeness and maximum principle}

We say that the weak maximum principle at infinity holds on a weighted manifold $M_{f}$ if for every $u \in C^{2}(M)$, with $\sup _{M} u=u^{*}<+\infty$, there exists a sequence $\left\{x_{k}\right\}$ along which

$$
\text { (i) } u\left(x_{k}\right)>u^{*}-\frac{1}{k}, \quad \text { (ii) } \Delta_{f} u\left(x_{k}\right)<\frac{1}{k} \text {. }
$$

It is now well known, [17], [18], that this principle is equivalent to the stochastic completeness of the diffusion process associated to $\Delta_{f}$. This means that the heat kernel of the $f$-Laplacian $p_{f}(x, y, t)$ satisfies the conservation property

$$
\int_{M} p_{f}(x, y, t) d \operatorname{vol}_{f}(y)=1,
$$

for every $x \in M$ and $t>0$. The $f$-stochastic completeness, in turn, is implied by the volume condition

$$
\frac{R}{\log \operatorname{vol}_{f}\left(B_{R}\right)} \notin L^{1}(+\infty) .
$$

For a complete weighted manifold $M_{f}$ satisfying $\operatorname{Ric}_{f} \geq \lambda$ we have, for constants $A, B$ and $C$, the Qian-Wei-Wylie volume estimate [23], [27]:

$$
\operatorname{vol}_{f}\left(B_{R}\right) \leq A+B \int_{R_{0}}^{R} e^{-\lambda t^{2}+C t} d t, \quad R>>1 .
$$

This implies that every complete gradient Ricci soliton is $f$-stochastically complete, and therefore satisfies the weak maximum principle for the $f$-Laplacian.

In fact, using estimates for the potential function due to Z.-H. Zhang, [28], that will be described in Section 5.1 below, and a general result contained in [19], one proves that on every gradient Ricci soliton, the full Omori-Yau maximum principle holds, both for the $f$-Laplacian and for the ordinary Laplacian. Namely,

Theorem 3.1. Let $(M,\langle\rangle$,$) be any gradient Ricci soliton. Then for every function$ $u \in C^{2}(M)$ bounded for above, there exists a sequence $\left\{x_{k}\right\}$ such that $u\left(x_{n}\right) \rightarrow u^{*}$, $\left|\nabla u\left(x_{k}\right)\right|<1 / k$ and $\Delta_{f} u\left(x_{k}\right)<1 / k\left(\operatorname{resp} . \Delta u\left(x_{k}\right)<1 / k\right)$.

We point out that the case of shrinking solitons was recently obtained by M. Fernández-López and E. García-Río, [10].

\section{The Feller property}

The weak maximum principle at infinity for the $f$-Laplacian is a powerful tool for deducing qualitative information about the solutions of differential inequalities of the form

$$
\Delta_{f} u \geq \Lambda(u) .
$$

See [17]. 
Accordingly, every solution $u$ of (4.1) bounded from above on the whole manifold $M$ satisfies

$$
\Lambda\left(u^{*}\right) \leq 0
$$

This fact has many applications in geometric analysis. Our purpose now is to investigate qualitative properties of solutions of (4.1) which are defined only in a neighborhood of infinity. This requires the introduction of new tools that can be developed under the validity of a further stochastic property of the underlying manifold, namely, the Feller property.

Note that if $M$ is stochastically complete for $\Delta_{f}$, then a bounded solution $u>0$ of the differential inequality

$$
\Delta_{f} u \geq \lambda u
$$

outside a smooth domain $\Omega \subset \subset M$ satisfies

$$
u(x) \leq c \cdot h(x) \quad \text { on } M \backslash \Omega,
$$

where $c>0$ is a suitable constant and $h>0$ is the minimal solution of the problem

$$
\left\{\begin{array}{cl}
\Delta_{f} h=\lambda h & \text { in } M \backslash \bar{\Omega} \\
h=1 & \text { on } \partial \Omega
\end{array}\right.
$$

(which is constructed by means of an exhaustion procedure).

Indeed, let $c=\sup _{\partial \Omega} u$. Then, for every $\epsilon>0$,

$$
\Delta_{f}(u-c h-\epsilon) \geq \lambda(u-c h) \geq \lambda(u-c h-\epsilon) \quad \text { on } M \backslash \bar{\Omega}
$$

and $u-c h-\epsilon \leq-\epsilon$ on $\partial \Omega$. Therefore the function $v_{\epsilon}=\max \{0, u-c h-\epsilon\}$ is bounded, nonnegative and satisfies $\Delta_{f} v_{\epsilon} \geq \lambda v_{\epsilon}$. Since $M$ is stochastically complete with respect to $\Delta_{f}, v_{\epsilon} \equiv 0$, that is, $u \leq c h+\epsilon$, and the conclusion follows by letting $\epsilon \rightarrow 0$.

In particular, if $h(x) \rightarrow 0$ as $x \rightarrow \infty$, we can deduce that the same holds for the original function $u$.

According to a characterization by R. Azencott [1], it happens that the required decay property of $h$ is equivalent to the Feller property on $M$ with respect to $\Delta_{f}$, that is, that the heat semigroup generated by $-\Delta_{f}$ maps the space $C_{o}(M)$ of continuous functions vanishing at infinity into itself, or equivalently, that for every relatively compact open set $\Omega$ in $M$, the heat kernel $p_{f}$ of $-\Delta_{f}$ satisfies

$$
\int_{\Omega} p_{f}(x, y, t) d \operatorname{vol}_{f}(y) \rightarrow 0 \quad \text { as } x \rightarrow+\infty .
$$

We thus obtain the following result:

Theorem 4.1. Let $M_{f}$ be $f$-stochastically complete. If $M_{f}$ is Feller, then every bounded solution $v>0$ of

$$
\Delta_{f} v \geq \lambda v \quad \text { on } M \backslash \bar{\Omega}
$$

satisfies

$$
v(x) \rightarrow 0 \quad \text { as } x \rightarrow \infty .
$$


On the basis of these observations, we prove the following theorem:

Theorem 4.2. Let $M_{f}$ be a stochastically complete and Feller manifold for $\Delta_{f}$. Consider the differential inequality

$$
\Delta_{f} u \geq \Lambda(u) \text {, on } M \backslash \Omega,
$$

where $\Omega \subset \subset M$ and the function $\Lambda:[0,+\infty) \rightarrow[0,+\infty)$ is either continuous or nondecreasing, and satisfies the following conditions:

$$
\text { (a) } \Lambda(0)=0 ; \quad \text { (b) } \Lambda(t)>0, \forall t>0 ; \quad \text { (c) } \liminf _{t \rightarrow 0+} \frac{\Lambda(t)}{t^{\xi}}>0
$$

for some $0 \leq \xi \leq 1$. Then, every bounded solution $u>0$ of (4.2) satisfies

$$
\lim _{x \rightarrow \infty} u(x)=0 .
$$

Proof. Let us consider the case where $f$ is not continuous. The other case is easier. By assumption, there exists $0<\varepsilon<1 / 2$ and $c>0$ such that

$$
\Lambda(t) \geq c t^{\xi} \quad \text { on }(0,2 \varepsilon) \text {. }
$$

Since $t^{\xi} \geq t$ on $(0,1]$, and $\Lambda$ is nondecreasing, then

$$
\Lambda(u(x)) \geq \Lambda_{\varepsilon}(u(x))= \begin{cases}c u, & \text { if } u(x)<\varepsilon \\ c \varepsilon, & \text { if } u(x) \geq \varepsilon .\end{cases}
$$

On the other hand, since $u>0$ is bounded, if we set $u^{*}=\sup _{M \backslash \Omega} u$, then

$$
c \varepsilon \geq \frac{c \varepsilon}{u^{*}} u^{*} \geq \frac{c \varepsilon}{u^{*}} u .
$$

It follows that

$$
\Delta_{f} u \geq \Lambda_{\varepsilon}(u) \geq \lambda u
$$

where

$$
\lambda=c \min \left\{1, \frac{\varepsilon}{u^{*}}\right\}>0 .
$$

Using the Feller property we now conclude that $u(x) \rightarrow 0$, as $x \rightarrow \infty$.

\section{Estimates for the $f$-Laplacian of the distance function and comparison results}

By comparison arguments and radialization techniques, many of the properties of solutions of differential in(equalities) involving the $f$-Laplacian, and in particular, the stochastic properties of $\Delta_{f}$ may be deduced by imposing suitable bounds on $\Delta_{f} r$ where $r(x)$ denotes the distance function from a reference point $o \in M$. We are going to collect some results along these lines concerning stochastic completeness, the Feller property, the Omori-Yau maximum principle and the compact support principle. Items (i) and (ii) in the next theorem are weighted versions of parts (c) and (d) of Corollary 15.2 in [12]. 
Theorem 5.1. Let $M_{f}$ be a weighted Riemannian manifold, and let $r(x)$ be the distance from a fixed point $o \in M$. Let also $g$ be a $C^{2}$ odd function on $\mathbb{R}$ satisfying $g(0)=0, g^{\prime}(0)=1$ and $g(t)>0$ for all $t>0$.

(i) Assume that there exist $R_{0} \geq 0$ and a constant $n>1$ such that for every $x$ within the cut locus of o with $r(x)>R_{0}$ we have

$$
\Delta_{f} r(x) \leq(n-1) \frac{g^{\prime}}{g}(r(x)) \quad \text { with } \quad \frac{\int_{0}^{r} g(t)^{n-1} d t}{g(r)^{n-1}} \notin L^{1}(+\infty) .
$$

Then $M_{f}$ is stochastically complete.

(ii) Assume that $o$ is a pole and that there exist $R_{0} \geq 0$ and a constant $n>1$ such that, for $r(x)>R_{0}$, we have

$$
\Delta_{f} r(x) \geq(n-1) \frac{g^{\prime}}{g}(r(x)) \quad \text { with } \quad \frac{\int_{0}^{r} g(t)^{n-1} d t}{g\left((r)^{n-1}\right.} \in L^{1}(+\infty) .
$$

Then $M_{f}$ is not stochastically complete.

(iii) Assume that $o$ is a pole and that there exist $R_{0} \geq 0$ and a constant $n>1$ such that for $r(x)>R_{0}$ we have

$$
\Delta_{f} r(x) \geq(n-1) \frac{g^{\prime}}{g}(r(x))
$$

and that either

$$
\frac{1}{g^{n-1}(r)} \in L^{1}(+\infty)
$$

or

$$
\text { (a) } \frac{1}{g^{n-1}(r)} \notin L^{1}(+\infty) \text { and } \quad \text { (b) } \frac{\int_{r}^{+\infty} g^{n-1}(t) d t}{g^{n-1}(r)} \notin L^{1}(+\infty) \text {. }
$$

Then $M_{f}$ is Feller.

(iv) Assume there exist $R_{0} \geq 0$ and a constant $n>1$ such that if $x$ is within the cut locus of o with $r(x)>R_{0}$ we have

$$
\Delta_{f} r(x) \leq(n-1) \frac{g^{\prime}}{g}(r(x))
$$

and that $g$ does not satisfy the conditions in (iii). Then $M_{f}$ is not Feller.

Proof. We outline the proof, which follows the lines of arguments valid for the ordinary Laplacian. To prove (i) and (ii), let $\alpha(r)$ be the function defined by

$$
\alpha(r)=\int_{0}^{r} \frac{\int_{0}^{t} g(s)^{n-1} d s}{g(t)^{n-1}} d t
$$


and note that

$$
\alpha^{\prime}(r)>0, \quad \alpha^{\prime \prime}(r)+(n-1) \frac{g^{\prime}}{g} \alpha^{\prime}(r)=1 .
$$

Let also $v(x)=\alpha(r(x))$, so that $v$ is $C^{2}$ within the cut locus of $o$ and there

$$
\Delta_{f} v(x)=\alpha^{\prime \prime}(r(x))+\Delta_{f} r(x) \alpha^{\prime}(r(x)) .
$$

Now, in case (ii), $v$ is $C^{2}$ and bounded from above on $M$, and since $\alpha^{\prime}>0$ it does not attain a maximum. Thus, if $R$ is large enough that (5.2) holds in $B_{R}^{c}$ and $\gamma<u^{*}$ is sufficiently close to $u^{*}$ that $\Omega_{\gamma}=\{x: u(x)>\gamma\} \subset B_{R}^{c}$, then, in $\Omega_{\gamma}$

$$
\Delta_{f} v \geq \alpha^{\prime \prime}(r(x))+(n-1) \frac{g^{\prime}}{g} \alpha^{\prime}(r(x))=1,
$$

so that $v$ violates the weak maximum principle at infinity. Therefore $M_{f}$ is not stochastically complete.

To prove (i), let $u$ be $C^{2}$ and bounded from above on $M$. We claim that for every $\gamma<u^{*}, \inf _{\Omega_{\gamma}} \Delta_{f} u \leq 0$ which clearly implies that the weak maximum principle at infinity holds for $\Delta_{f}$ and $M_{f}$ is stochastically complete.

Note that in the present situation $v$ tends to infinity as $r(x) \rightarrow \infty$ and satisfies

$$
\Delta_{f} v \leq \alpha^{\prime \prime}+(n-1) \frac{g^{\prime}}{g} \alpha^{\prime} \leq 1
$$

for $x$ within the cut locus of $o$ and such that $r(x)>R_{0}$.

Assume, aiming at a contradiction, that there exists $\gamma<u^{*}$ such that

$$
\inf _{\Omega_{c}} \Delta_{f} u \geq 2 c>0 .
$$

Clearly $u$ does not attain its supremum, and, by taking $\gamma$ close enough to $u^{*}$, we may arrange that (5.1) holds on $\Omega_{\gamma}$. Let $x_{o} \in \Omega_{\gamma}$ and choose $0<\delta<c$ small enough that the function $\tilde{u}=u-\gamma-\delta v$ is positive at $x_{o}$. Since $\tilde{u}<0$ on $\Omega_{\gamma}$, and tends to $-\infty$ as $r(x) \rightarrow+\infty$, it attains a positive maximum at $\bar{x} \in \Omega_{\gamma}$, and using the Calabi trick we may assume that $r(x)$ is smooth at $\bar{x}$. Then, at $\bar{x}$,

$$
\Delta_{f} \tilde{u}(\bar{x})=\Delta_{f} u(\bar{x})-\delta \Delta_{f} v(\bar{x}) \geq 2 c-\delta \geq c>0,
$$

which yields the required contradiction.

We now come to the Feller property. In case (iii), the conditions satisfied by $g$ imply that the model manifold $M_{g}^{n}$ defined as $\mathbb{R}^{n}$ endowed with the metric

$$
\langle,\rangle=d r^{2}+g(r)^{2} d \theta^{2},
$$

is Feller (with respect to the ordinary Laplacian), and, according to Theorem 4.4 and Lemma 5.1 in [20], the radial minimal solution $\beta$ of the exterior boundary value problem

$$
\left\{\begin{array}{l}
\beta^{\prime \prime}+(n-1) \frac{g^{\prime}}{g} \beta^{\prime}=\lambda \beta \\
\beta\left(R_{0}\right)=1
\end{array}\right.
$$


tends to 0 as $r$ tends to infinity and satisfies $h^{\prime}(r)<0$. As above, let us define $v(x)=\beta(r(x))$, so that $v(x) \rightarrow 0$ as $r(x) \rightarrow \infty$ and

$$
\Delta_{f} v=\beta^{\prime \prime}+\Delta_{f} r \beta^{\prime} \leq \beta^{\prime \prime}+(n-1) \frac{g^{\prime}}{g} \beta^{\prime}=\lambda v .
$$

For every $R>R_{0}$, let $h_{R}$ be the solution of the exterior boundary value problem

$$
\left\{\begin{aligned}
\Delta_{f} h_{R} & =\lambda h_{R}, \\
h_{R}\left(R_{0}\right) & =1, h_{R}(R)=1,
\end{aligned}\right.
$$

and note that by the comparison principle $h_{R}<v$ in $B_{R} \backslash B_{R_{0}}$. As $R \rightarrow+\infty, h_{R}$ tends to the minimal solution $h$ of the problem

$$
\left\{\begin{aligned}
\Delta_{f} h & =\lambda h, \\
h\left(R_{0}\right) & =1 .
\end{aligned}\right.
$$

Since clearly $0<h<v$ in $M \backslash B_{R_{0}}$, $h$ tends to 0 as $r(x)$ tends to $\infty$ and $M_{f}$ is Feller.

Finally, assume that (5.6) holds and that $g$ does not satisfy the conditions in (iii). Note that in particular, $g^{-n+1} \notin L^{1}(+\infty)$ while $g^{n-1} \in L^{1}(+\infty)$, and according to (i) $M_{f}$ is stochastically complete. By Theorem 4.4 of [20], the model manifold $M_{g}^{n}$ is not Feller, and therefore the minimal radial solution $\beta$ of the exterior problem (5.7) does not tend to zero as $r \rightarrow+\infty$. Since $\beta^{\prime} \leq 1$ by Corollary 5.1 of [20], it follows that the function $v(x)=\beta(r(x))$ is a bounded solution of

$$
\Delta_{f} v \geq \lambda v
$$

which satisfies $v=1$ on $R_{0}$ and which does not tend to zero at infinity. It follows from the discussion preceding Theorem 4.1 that the minimal solution of the exterior problem (5.8) satisfies $v \leq c h$, for some constant $c>0$, so that $h$ does not tend to zero at infinity and $M_{f}$ is not Feller.

Remark 5.2. In the case that $n$ is an integer, the differential inequalities satisfied by $\Delta_{f} r$ can be interpreted as comparison with the Laplacian of the distance function of the model $M_{g}^{n}$. It is also interesting to observe that in the case of the Feller property the inequalities assumed for $\Delta_{f} r$ go in the opposite direction than those assumed in the case of stochastic completeness.

In the case of the ordinary Laplacian, upper and lower estimates for $\Delta r$ may be obtained via the Laplacian comparison theorem imposing lower bounds on the Ricci curvature, or upper bounds on the sectional curvature, respectively. In the case of the $f$-Laplacian, there does not seem to be an analogue of the sectional curvature whose control allows to obtain lower estimates for $\Delta_{f} r$. As for upper estimates, the most effective way to obtain upper bounds for $\Delta_{f} r$ is to impose lower bounds on the modified Bakry-Emery Ricci tensor

$$
\operatorname{Ric}_{\alpha}=\operatorname{Ric}+\operatorname{Hess} f-\frac{1}{\alpha} d f \otimes d f
$$


with $\alpha>0$, but in view of applications to Ricci solitons it is important to try and obtain estimates for $\Delta_{f} r$ assuming a lower bound on the Bakry-Emery Ricci tensor $\operatorname{Ric}_{f}$ corresponding to $\alpha=+\infty$, together with some control on the weight $f$ or its gradient.

Indeed, as Theorem 2.1 of Quian's [23] shows, in the more general case of operators of the form $\Delta+X$ with a drift which is not necessarily a gradient, that upper bounds for $\Delta_{f} r$ follow from imposing lower bounds on Ric $f$ and a control on the drift term $X$. More precisely we have:

Theorem 5.3. Let $M_{f}$ be a weighted manifold, let o be a reference point in $M$ and let $r(x)=d(x, 0)$ be the Riemannian distance from $o$.

(i) Assume that

$$
\operatorname{Ric}_{f} \geq-k^{2}
$$

for some constant $k \geq 0$. Then there exists a constant $C$ depending only on $M$, on $\nabla f$ and on o such that

$$
\Delta_{f} r(x) \leq C+\frac{m-1}{r(x)}+k^{2} \cdot r(x) \quad \text { on } M \backslash \operatorname{cut}(o)
$$

(ii) Assume that

$$
\left\{\begin{array}{l}
\operatorname{Ric}_{f}(x) \geq-k_{1}^{2}(r(x)) \\
|\nabla f|(x) \leq k_{2}(r(x))
\end{array}\right.
$$

where $k_{i}(r)$ are continuous nondecreasing functions satisfying $k_{i}(r) \rightarrow+\infty$ as $r \rightarrow+\infty$. Then

$$
\Delta_{f} r(x) \leq m \frac{g^{\prime}(r(x))}{g(r(x)} \quad \text { on } M \backslash \operatorname{cut}(o),
$$

where $g:[0,+\infty) \rightarrow[0,+\infty)$ is the solution of the initial value problem

$$
\left\{\begin{array}{l}
g^{\prime \prime}(r)-\frac{k_{1}(r)^{2}+k_{2}(r)^{2}}{m} g(r)=0, \\
g(0)=0, g^{\prime}(0)=1
\end{array}\right.
$$

(iii) Assume that there are nonnegative constants $k$ and $C$ such that

$$
\left\{\begin{array}{l}
\operatorname{Ric}_{f} \geq-k, \\
|\nabla f| \leq C(d(x, o)+1) \quad \forall x \in M .
\end{array}\right.
$$

For every $p \in M$, and every $x \in M \backslash(\{p\} \cup \operatorname{cut}(p))$, if $\rho(x)=d(x, p)$, then

$$
\Delta_{f} \rho(x) \leq \frac{m-1}{\rho}+\frac{1}{3}(k+2 C) \rho+C(1+d(o, p)) .
$$

Applying Theorem 5.1 we obtain a version for $\Delta_{f}$ of Theorems 1.5 and 1.6 of Qian ([23]) on the stochastic completeness of Laplacians with drift. We point out that the proof we present is entirely deterministic. 
Theorem 5.4. Let $M_{f}$ be a complete weighted Riemannian manifold, and assume that either

$$
\operatorname{Ric}_{f} \geq-k^{2} \quad \text { for some constant } k \geq 0 \text {, }
$$

or

$$
\left\{\begin{array}{l}
\operatorname{Ric}_{f}(x) \geq-k_{1}^{2}(r(x)) \\
|\nabla f|(x) \leq k_{2}(r(x))
\end{array}\right.
$$

where $k_{i}(r)$ are continuous nondecreasing functions satisfying $k_{i}(r) \rightarrow+\infty$ as $r \rightarrow+\infty$ and

$$
\frac{1}{\sqrt{k_{1}^{2}(t)+k_{2}^{2}(t)}} \notin L^{1}(+\infty) .
$$

Then $M_{f}$ is stochastically complete for $\Delta_{f}$.

Proof. If (5.10) holds, then $\Delta_{f} r$ satisfies the estimate (i) in Theorem 5.3, and it is easily seen that this implies (5.1) with $n=m$ and

$$
g(r)=r \exp \left[\frac{1}{m-1}\left(C r+\frac{k}{2} r^{2}\right)\right]
$$

and it is clear that $g(r)$ satisfies the nonintegrability in (5.1), and $M_{f}$ is then stochastically complete by Theorem 5.1 (i).

Assuming that (5.11) and (5.12) hold, then $\Delta_{f} r(x)$ satisfies the estimate in Theorem 5.3 (ii) with $g$ a solution of (5.9). This implies that $\psi=g^{\prime} / g$ satisfies the Riccati equation

$$
\psi^{\prime}+\psi^{2}=k^{2}, \quad \text { with } k(r)^{2}=\frac{k_{1}(r)^{2}+k_{2}(r)^{2}}{m},
$$

and $\psi(r)=1 / r+O(1)$ as $r \rightarrow 0$. Thus $\psi^{\prime}<0$ whenever $\psi>k$ and since $k(r)$ is increasing and tends to $+\infty$ as $r \rightarrow+\infty$, standard arguments show that there exists $r_{o}$ such that $\psi\left(r_{o}\right)=k\left(r_{o}\right)$ and $\psi(r) \leq k(r)$ for all $r \geq r_{o}$.

This in turn implies that there exists $R$ sufficiently large and a constant $C>0$ such that

$$
\frac{\int_{0}^{r} g(t)^{m} d t}{g(r)^{m}} \geq \frac{C}{k(r)} \quad \text { if } r \geq R .
$$

Indeed, $g(r) \rightarrow+\infty$ as $r \rightarrow+\infty$ and, using L'Hôpital's rule, and the fact that $k$ is nondecreasing we have

$$
\liminf _{r \rightarrow \infty} \frac{k(r) \int_{0}^{r} g(t)^{m} d t}{g(r)^{m}} \geq \liminf _{r \rightarrow \infty} \frac{k(r) g(r)}{m g^{\prime}(r)} \geq \frac{1}{m} .
$$

By $(5.12), 1 / k(r) \notin L^{1}(+\infty)$ and, again by Theorem 5.1 (i), $M_{f}$ is stochastically complete. 
We stress that, in order to deduce the validity of the Feller property using Theorem 5.1 (iii), one needs a control from below on $\Delta_{f} r$, while lower bounds on $\operatorname{Ric}_{f}$ typically produce upper bounds on $\Delta_{f} r$.

Using a probabilistic technique which extends to the Laplacian with a drift term a previous result of $\mathrm{P}$. Hsu $([14])$ for $\Delta$, and which essentially consists in genuine estimates on hitting time probabilities, Qian (see Theorem 1.7 of [23]) proves the following theorem. Note that, again, Qian's result actually applies to the more general operators of the form $L=\Delta+X$.

Theorem 5.5. Let $M_{f}$ be a complete weighted manifold and assume that for some $o \in M$ the conclusion of Theorem 5.3 (iii) holds, namely, there exist constants $k$ and $C>$ such that, for every $p \in M$, if $\rho(x)=d(x, p)$ denotes the distance from $p$, we have

$$
\Delta_{f} \rho(x) \leq \frac{m-1}{\rho}+\frac{1}{3}(k+2 C) \rho+C(1+d(o, p)) .
$$

Then $M_{f}$ is Feller with respect to $\Delta_{f}$. In particular, if $\operatorname{Ric}_{f}$ and $\nabla f$ satisfy the estimates in Theorem 5.3 (iii), then $M_{f}$ is Feller with respect to $\Delta_{f}$.

We next describe a result which extends a condition on the validity of the full Omori-Yau maximum principle for the operator $\Delta_{f}$ proved in [19]. The argument we use is an adaptation of a recent elegant proof of the Omori-Yau maximum principle due to A. Borbély, [5] and [6]. We are grateful to A. Borbély for sending us a copy of [6].

Theorem 5.6. Let $M_{f}$ be a complete weighted manifold and assume that there exists a nonnegative $C^{2}$ function $\gamma$ satisfying the following conditions:

$$
\begin{aligned}
& \gamma(x) \rightarrow+\infty \text { as } x \rightarrow \infty \\
& \exists A>0 \text { such that }|\nabla \gamma| \leq A \text { off a compact set; } \\
& \exists B>0 \text { such that } \Delta_{f} \gamma \leq B G(\gamma) \text { off a compact set, }
\end{aligned}
$$

where $G$ is a smooth function on $[0,+\infty)$ satisfying

$$
\text { (i) } G(0)>0 \quad \text { (ii) } G^{\prime}(t) \geq 0 \text { on }[0,+\infty)
$$

(iii) $G(t)^{-1} \notin L^{1}(+\infty)$.

Then the Omori-Yau maximum principle holds for $\Delta_{f}$. The same conclusion holds if $\gamma(r)=r(x)$ is the distance from a reference point $o$ and we assume that (5.15) holds in the complement of the cut locus of o (while of course (5.13) and (5.14) are automatically satisfied).

Proof. We outline the proof, which follows Borbely's argument closely. Let $u$ be a $C^{2}$ function such that $u^{*}=\sup _{M} u<+\infty$. We want to show that there exists a sequence $\left\{x_{n}\right\}$ such that $u\left(x_{n}\right) \rightarrow u^{*}, \lim _{n}\left|\nabla u\left(x_{n}\right)\right|=0$ and $\lim \sup _{n} \Delta_{f} u\left(x_{n}\right) \leq 0$. We may assume that $u$ does not attain its supremum, for otherwise the conclusion is obvious. Define

$$
F(t)=\exp \left\{\int_{0}^{t} G(s)^{-1} d s\right\},
$$


so that $F$ is nondecreasing and $\lim _{t \rightarrow+\infty} F(t)=+\infty$. For every $t$, let

$$
\Omega_{t}=\{x: \gamma(x)>t\} .
$$

Since $\gamma$ is proper, there exists $t_{o}$ such that the inequalities (5.14) and (5.15) hold in the set $\Omega_{t_{o}}$. Let $\epsilon<\min \left\{1, u^{*}-\sup _{\Omega_{t_{o}}^{c}} u\right\}$, and define $h_{\lambda}(x)=\lambda F(\gamma(x))+u^{*}-\epsilon$. Since $F \geq 1$, if $\lambda>\epsilon$ then $h_{\lambda}>u^{*}>u$ on $M$. Let $\lambda_{o}=\inf \left\{\lambda: h_{\lambda}(x)>\right.$ $u(x) \forall x \in M\}$, and note that since $u<u^{*}$ on $M$ then $\lambda_{o}>0$, and, by continuity, $h_{\lambda_{o}}(x) \geq u(x)$ for every $x$ in $M$.

We claim that there exists $x_{\epsilon}$ such that $h_{\lambda_{o}}\left(x_{\epsilon}\right)=u\left(x_{\epsilon}\right)$. Note that since $h_{\lambda_{o}}>u^{*}-\epsilon>\sup _{\Omega_{t_{o}}^{c}} u, x_{\epsilon}$ necessarily lies in $\Omega_{t_{o}}$.

To prove the claim, we will show that if $h_{\lambda}>u$ on $M$ for some $\lambda>0$ then there exists $\lambda^{\prime}<\lambda$ such that $h_{\lambda^{\prime}}>u$ on $M$. Indeed, since $F(\gamma) \rightarrow+\infty$ as $\gamma \rightarrow+\infty$, and $\gamma$ is proper, there exists $t_{1}>t_{o}$ such that $h_{\lambda}>u^{*}+1$ in $\Omega_{t_{1}}$. Since $\Omega_{t_{1}}^{c}$ is compact, and $h_{\lambda}(x)>u(x)$ on $M$, we may choose $\lambda^{\prime}<\lambda$ sufficiently close to $\lambda$ so that $h_{\lambda^{\prime}}>u$ on $\Omega_{t_{1}}^{c}$, and $h_{\lambda^{\prime}}>u^{*}$ on $\partial \Omega_{t_{1}}$. Since $F$ is increasing, $h_{\lambda^{\prime}}>u^{*}$ in $\Omega_{t_{1}}$ and therefore $h_{\lambda^{\prime}}>u$ on $M$, as required.

Next we claim that $h_{\lambda_{o}}$ is smooth at $x_{\epsilon}$. This is clear if $\gamma$ is $C^{2}$ on $M$, while if $\gamma(x)=r(x)$ is the Riemannian distance function, the proof in Borbely's paper, which only uses the fact that $u-h_{\lambda_{o}}$ attains a maximum at $x_{\epsilon}$, and properties of the function $r(x)$, applies without changes.

Thus, since $u\left(x_{\epsilon}\right)=h_{\lambda_{o}}\left(x_{\epsilon}\right)=\lambda_{o} F\left(\gamma\left(x_{\epsilon}\right)\right)+u^{*}-\epsilon<u^{*}=\sup u$ we have

$$
u\left(x_{\epsilon}\right)>u^{*}-\epsilon \text { and } \lambda_{o} F\left(\gamma\left(x_{\epsilon}\right)\right)<\epsilon .
$$

Also, since $u-h_{\lambda_{o}}$ attains a maximum at $x_{\epsilon}$,

(i) $\nabla u\left(x_{\epsilon}\right)=\lambda_{o} F^{\prime}\left(\gamma\left(x_{\epsilon}\right)\right) \nabla \gamma\left(x_{\epsilon}\right)$

(ii) $\Delta_{f} u\left(x_{\epsilon}\right) \leq \Delta_{f} h_{\lambda_{o}}\left(x_{\epsilon}\right)=\lambda_{o}\left[F^{\prime \prime}\left(\gamma\left(x_{\epsilon}\right)\right)\left|\nabla \gamma\left(x_{\epsilon}\right)\right|^{2}+F^{\prime}\left(\gamma\left(x_{\epsilon}\right)\right) \Delta_{f} \gamma\left(x_{\epsilon}\right)\right]$.

Easy computations show that $F^{\prime}=F / G$ and $F^{\prime \prime} \leq F / G^{2}$ so that (i) above and (5.14) yield

$$
\left|\nabla u\left(x_{\epsilon}\right)\right| \leq A \lambda_{o} \frac{F\left(\gamma\left(x_{\epsilon}\right)\right)}{G\left(\gamma\left(x_{\epsilon}\right)\right)}<\frac{A}{G(0)} \epsilon,
$$

while using (ii) and (5.15) we get

$$
\Delta_{f} u\left(x_{\epsilon}\right) \leq \lambda_{o} F\left(\gamma\left(x_{\epsilon}\right)\right)\left[\frac{1}{G^{2}\left(\gamma\left(x_{\epsilon}\right)\right)}+\frac{\Delta_{f} u\left(x_{\epsilon}\right)}{G\left(\gamma\left(x_{\epsilon}\right)\right)}\right] \leq(1+B) \lambda_{o} F\left(x_{\epsilon}\right)<(1+B) \epsilon
$$

We conclude this section with a brief discussion about the compact support principle for the operator $\Delta_{f}$ (see [21] and [22], where more general quasilinear elliptic operators in divergence form are considered).

A function $u$ is said to be a semiclassical solution of the differential inequality

$$
\Delta_{f} u \geq \lambda(u)
$$


in a domain $\Omega$ if $u \in C^{1}(\Omega)$ and $u$ satisfies the inequality in weak sense, that is

$$
\int_{\Omega}[\langle\nabla f, \nabla \phi\rangle+\lambda(u) \phi] \leq 0
$$

for every nonnegative $\phi \in C_{c}^{1}(\Omega)$.

The compact support principle is said to hold for the differential inequality (5.17) if, whenever $\Omega$ is an exterior domain, namely $\Omega \supset M \backslash B_{R}(o)$ for some $R>0$, and $u \geq 0$ is a semiclassical solution of (5.17) in $\Omega$ with the property that $u(x) \rightarrow 0$ as $r(x) \rightarrow+\infty$ that $u$ vanishes identically outside a compact set.

We are grateful to M. Rigoli for pointing that the validity of the compact support principle depends essentially on the properties of the operator and of the function $\lambda$, and that the effect of the geometry is once again encoded by the behavior of $\Delta_{f} r(x)$.

Indeed, the proof of Theorem 1.1 in [22], may be adapted with minor changes to obtain the following:

Theorem 5.7. Let $M_{f}$ be a complete weighted manifold, and let $\lambda$ be a continuous function on $[0,+\infty)$ which vanishes in 0 and is nondecreasing and strictly positive on some interval $(0, \delta), \delta>0$. Set $\Lambda(t)=\int_{0}^{t} \lambda(s) d s$. If

$$
\frac{1}{\sqrt{\Lambda(s)}} \in L^{1}(0+),
$$

and there exists $C>0$ such that the differential inequality

$$
\Delta_{f} r(x) \geq-C^{2}>-\infty
$$

holds weakly on $M$, then the compact support principle holds for the differential inequality (5.17).

As an immediate consequence, as noted in [22], if (5.18) holds, $\Delta_{f}=\Delta$, and $M$ is a Cartan-Hadamard manifold, then the compact support principle holds for $(5.17)$.

\subsection{Feller property for Ricci solitons}

In this section we are going to prove that Ricci solitons are Feller with respect to the weighted Laplacian $\Delta_{f}$ and with respect to the ordinary Laplacian $\Delta$.

The fact that for every gradient Ricci soliton the weighted Laplacian $\Delta_{f}$ is Feller is then a consequence of the results of the previous section and of the following estimates for the gradient of the potential function which have been obtained by Zhang, [28] (see also H.-D. Cao and D. Zhou, [9]). These in turn depend on lower estimates for the scalar curvature of Ricci solitons obtained by Zhang, and by a different method in [18]. We are going to briefly describe the latter approach. We recall from [18] the following a-priori estimate for weak solutions of semilinear elliptic inequalities under volume assumptions (see also Theorem B in [16]). 
Theorem 5.8. Let $\left(M,\langle\rangle,, e^{-f} d \mathrm{vol}\right)$ be a complete, weighted manifold. Let $a(x)$, $b(x) \in C^{0}(M)$, set $a_{-}(x)=\max \{-a(x), 0\}$ and assume that

$$
\sup _{M} a_{-}(x)<+\infty \quad \text { and } \quad b(x) \geq \frac{1}{Q(r(x))} \quad \text { on } M,
$$

for some positive, nondecreasing function $Q(t)$ such that $Q(t)=o\left(t^{2}\right)$, as $t \rightarrow+\infty$. Assume furthermore that, for some $H>0$,

$$
\frac{a_{-}(x)}{b(x)} \leq H \quad \text { on } M .
$$

Let $u \in \operatorname{Lip}_{\text {loc }}(M)$ be a nonnegative weak solution of

$$
\Delta_{f} u \geq a(x) u+b(x) u^{\sigma},
$$

on $\left(M, e^{-f} d \mathrm{vol}\right)$, with $\sigma>1$. If

$$
\liminf _{r \rightarrow+\infty} \frac{Q(r) \log \operatorname{vol}_{f}\left(B_{r}\right)}{r^{2}}<+\infty,
$$

then

$$
u(x) \leq H^{\frac{1}{\sigma-1}} \quad \text { on } M .
$$

Using the volume estimate (3.1) we deduce the validity of the next:

Corollary 5.9. Let $(M,\langle\rangle,, \nabla f)$ be a complete Ricci soliton. Then the scalar curvature $S$ of $M$ satisfies the lower estimate

$$
S(x) \geq \begin{cases}0 & \text { if } \lambda \geq 0, \\ m \lambda & \text { if } \lambda<0 .\end{cases}
$$

Proof. Indeed,

$$
\Delta_{f} S=\lambda S-|\mathrm{Ric}|^{2} .
$$

and since $\mid$ Ric $\left.\right|^{2} \geq S^{2} / 2$, (Cauchy-Schwarz inequality), we have that the function $S_{-}(x)=\min \{0,-S(x)\}$ is a weak solution of the differential inequality

$$
\Delta_{f} S_{-} \geq \lambda S_{-}+\frac{1}{m} S_{-}^{2} .
$$

On the other hand, by (3.1), the condition (5.21) above is satisfied, and with an application of Theorem 5.8 with $a(x)=\lambda$ and $b(x)=1 / m$ shows that

$$
S_{-} \leq m \lambda_{-}
$$

and the conclusion follows.

Using these estimates in the basic equation

$$
S+|\nabla f|^{2}-2 \lambda f=C
$$

and integrating it along minimizing geodesics one obtains the estimates for the potential function and its gradient described in [28] and [9]. 
Lemma 5.10. Let $(M,\langle\rangle,, \nabla f)$ be a complete Ricci soliton. Then there exist positive constants $a$ and $b$ depending only on the soliton such that

$$
|\nabla f| \leq b+|\lambda| d(x, o) \quad \text { and } \quad|f(x)| \leq a+b d(x, o)+\frac{|\lambda|}{2} d(x, o)^{2} .
$$

Using these results we deduce the following:

Proposition 5.11. Let $(M,\langle\rangle,, \nabla f)$ be a complete Ricci soliton. Then $\Delta_{f}$ and $\Delta$ are both stochastically complete and Feller.

Proof. Since $\operatorname{Ric}_{f}=\operatorname{Ric}+\operatorname{Hess} f=\lambda g$ by definition, and

$$
|\nabla f| \leq C_{1}+|\lambda| d(x, o)
$$

by the lemma above, the conditions of Theorem 5.5 are satisfied and the statement concerning the $f$-Laplacian $\Delta_{f}$ follows.

In fact, as mentioned above, the above conditions imply that, if $p \in M$ and $\rho(x)=d(x, p)$ then $\Delta_{f} \rho$ satisfies the estimate

$$
\Delta_{f} \rho \leq \frac{m-1}{\rho}+C_{1} \rho+C_{2}(1+d(o, p)) .
$$

On the other hand

$$
|\langle\nabla f, \nabla \rho\rangle| \leq|\nabla f| \leq b+|\lambda| d(o, x)) \leq b+|\lambda|(d(+, p)+\rho(x))
$$

so that

$$
\Delta \rho=\Delta_{f} \rho+\langle\nabla f, \nabla \rho\rangle \leq \frac{m-1}{\rho}+\left(C_{1}+|\lambda|\right) \rho+\left(C_{2}+b+|\lambda|\right)(1+d(o, p))
$$

and, again by Theorem 5.5 the Laplacian $\Delta$ is Feller.

The estimates for the potential function described above allow us to prove Theorem 3.1.

Proof of Theorem 3.1. Both statements follow by applying Theorem 5.6 with the choice $\gamma(x)=r(x)^{2}$ and using the estimates for $\nabla f$ described above together with Qian's estimates for $\Delta_{f} r$ and $\Delta r$ as in the proof of Proposition 5.11.

\subsection{Applications of the Feller property to geometry and PDEs}

As alluded to at the beginning of Section 4, and formalized in Theorem 4.2, using the Feller property on a stochastically complete manifold enables one to extend the investigation of qualitative properties of solution of PDEs to the case where these are defined only in a neighborhood at infinity. In this section, we will illustrate the use of this viewpoint in a number of different geometric and analytic settings. We stress that the needed stochastic completeness assumptions are satisfied for a very rich family of examples. For instance, according to Proposition 5.11, a natural and 
important framework is given by Ricci solitons. In the non-weighted setting, we have the class of complete manifolds such that Ric $\geq-G(r)$, where $G(r)>0$ is a smooth increasing function satisfying $1 / \sqrt{G} \notin L^{1}(+\infty)$; [14]. Another admissible category for the application of Theorem 4.2 is given by Cartan-Hadamard manifolds with at most quadratic exponential volume growth. Actually, the (radial) sectional curvature assumption can be considerably relaxed as explained in Theorem 5.9 of [20].

5.2.1. Isometric immersions. Recall that if a Riemannian manifold $(M,\langle\rangle$, is stochastically complete, then the mean curvature $\mathbf{H}$ of a bounded isometric immersion $f: M \rightarrow \mathbb{B}_{R}(0) \subset \mathbb{R}^{n}$ must satisfy

$$
\sup _{M}|\mathbf{H}| R \geq 1
$$

In particular, a stochastically complete minimal submanifold in Euclidean space is necessarily unbounded. The next result show that this can be extended to the case where we have an isometric immersion of an end $E$ of $M$ with respect to a given compact subset $K$ of $M$. We observe that the concepts of stochastic completeness and the Feller property can be localized on one end $E$ simply requiring that its double $\mathcal{D}(E)$ be stochastically complete or Feller, respectively. It is then easy to verify that the maximum principle at infinity holds for every function $f: E \rightarrow \mathbb{R}$ which is bounded from above and does not attain its supremum on $\partial E$. It is easily verified that $M$ is stochastically complete if and only if so are all ends.

Similarly, the end $E$ is Feller if and only if for one (and therefore for all) relatively compact domain with smooth boundary $\Omega \supset K$, the minimal positive solution of the boundary value problem

$$
\left\{\begin{array}{cl}
\Delta h=\lambda h & \text { in } M \backslash \bar{\Omega}, \\
h=1 & \text { on } \partial \Omega,
\end{array}\right.
$$

tends to zero as $x \rightarrow \infty$ within the given end. Again, it is easy to see that an end $E$ satisfies this condition if and only if its double is Feller (see Section 7 of [20] for details).

We are now ready to state our result. We are grateful to R. Haslhofer and T. Ilmanen for helpful comments related to the formulation of the theorem.

Theorem 5.12. Let $(M,\langle\rangle$,$) be a Riemannian manifold and let E$ be an end of $M$ with respect to a compact set $K$. Assume that $E$ is stochastically complete and Feller, and that there exists a bounded isometric immersion $f: M \rightarrow \mathbb{B}_{R}(0) \subset \mathbb{R}^{n}$. Then the mean curvature of $f$ satisfies

$$
\sup _{E}|\mathbf{H}| R \geq 1
$$

Proof. Assume, aiming at a contradiction, that

$$
\sup _{E}|\mathbf{H}| R<1 .
$$


Straightforward computations show that $u=|f|^{2} \geq 0$ satisfies

$$
\Delta u \geq c \text { on } E
$$

where we have set

$$
c=2 m\left(1-\sup _{E}|\mathbf{H}| R\right)>0,
$$

and $m=\operatorname{dim}(M)$. If follows that $\Delta u \geq \lambda u$ on $E$ with $\lambda=c / R$.

Now, let $\Omega$ be a bi-collared relatively compact neighborhood of $\partial E$ in $\mathcal{D}(E)$. We use the same letters $f$ and $u$ to denote the obvious extensions of $f$ and $u$ to $\mathcal{D}$. We clearly have $f(\mathcal{D}(E) \backslash \Omega) \subset \mathbb{B}_{R}(0)$ and

$$
\Delta u \geq \lambda u \text { on } \mathcal{D}(E) \backslash \Omega .
$$

An application of Theorem 4.2 shows that $u(x) \rightarrow 0$, that is, $f(x) \rightarrow 0$ as $x \rightarrow+\infty$ in $M$. On the other hand, since strict inequality holds in $(5.25)$, for $R^{\prime}>R$ sufficiently close to $R$ we have $\sup _{E}|\mathbf{H}| R^{\prime}<1$, and clearly $f(E) \subset \mathbb{B}_{R^{\prime}}\left(0^{\prime}\right)$ provided $\left|0^{\prime}-0\right|<R^{\prime}-R$. Thus repeating the argument with $u^{\prime}(x)=\left|f(x)-0^{\prime}\right|^{2}$ we have

$$
\Delta u^{\prime} \geq c
$$

with the same value $c$, and then $u^{\prime}(x) \rightarrow 0$, i.e., $f(x) \rightarrow 0^{\prime} \neq 0$, as $x \rightarrow \infty$. This yields the required contradiction and the theorem is proved.

5.2.2. Conformal deformations. Given a Riemannian $m$-manifold $(M,\langle\rangle$,$) ,$ $m \geq 3$, consider the conformally related metric $\overline{\langle,\rangle}=v^{4 /(m-2)}\langle$,$\rangle , where v>0$ is a smooth function. The conformal factor $v$ obeys the Yamabe equation

$$
c_{m}^{-1} \Delta v-S v=-\bar{S} v^{\frac{m+2}{m-2}},
$$

where $S$ and $\bar{S}$ denote the scalar curvatures of $\langle$,$\rangle and \overline{\langle,\rangle}$, respectively. Assume that $M$ is stochastically complete and that

$$
\sup _{M} S(x) \leq S^{*} \text { and } \inf _{M} \bar{S}(x) \geq \bar{S}_{*}
$$

for some constants $S^{*} \geq 0$ and $\bar{S}_{*}>0$. An application of the weak minimum principle at infinity to the Yamabe equation shows that

$$
\left(\frac{S^{*}}{\bar{S}_{*}}\right)^{(m-2) / 4} \geq v_{*}=\inf _{M} v .
$$

In particular, if $S(x) \leq 0$ on $M$, then $v_{*}=0$. Actually, since the infimum of $v$ cannot be attained,

$$
\inf _{M \backslash \Omega} v=0,
$$

for every $\Omega \subset \subset M$. Clearly, to reach these conclusions the scalar curvature bound must hold on $M$. 
Theorem 5.13. Let $(M,\langle\rangle$,$) be a stochastically complete, Feller m-manifold,$ $m \geq 6$, with scalar curvature satisfying

$$
\sup _{M \backslash \Omega} S(x) \leq 0
$$

for some compact domain $\Omega \subset M$. Let $\overline{\langle,\rangle}=v^{4 /(m-2)}\langle$,$\rangle be a conformally related$ metric such that

$$
\inf _{M} v=v_{*}>0 .
$$

If the scalar curvature of $(M, \overline{\langle,\rangle})$ satisfies

$$
\inf _{M \backslash \Omega} \bar{S}(x)=\bar{S}_{*}>0,
$$

then

$$
v(x) \rightarrow+\infty \text { as } x \rightarrow \infty \text {. }
$$

With respect to the assumption that $S$ is nonnegative at infinity, one may wonder if it could be made nonnegative everywhere on $M$ with a conformal change of metric. Note, however, this in general would require control of the positive part $S^{+}$of $S$ in the set $\Omega$, which moreover may depend on the metric itself in a rather implicit way (see, e.g., Proposition 1.2 in [24]).

Proof. Just note that the positive, bounded function $u(x)=v(x)^{-1}$ satisfies

$$
c_{m}^{-1} \Delta u \geq-S u+\bar{S} u^{\frac{m-6}{m-2}} \geq \bar{S} u^{\frac{m-6}{m-2}} .
$$

Since

$$
0 \leq \frac{m-6}{m-2}<1
$$

Theorem 4.2 yields

$$
u(x) \rightarrow 0 \quad \text { as } x \rightarrow \infty .
$$

As an immediate consequence, we obtain the following non-existence result. Note that this applies, for instance, to an expanding, gradient Ricci soliton $M$. Indeed, in this case, the scalar curvature assumption is compatible with the restriction $\inf _{M} S \leq 0$ imposed by the soliton structure.

Corollary 5.14. On a stochastically complete and Feller m-manifold $(M,\langle\rangle$, with $m \geq 6$ and

$$
\sup _{M \backslash \Omega} S(x) \leq 0
$$

there is no conformal change $\overline{\langle,\rangle}=v^{4 /(m-2)}\langle$,$\rangle such that$

$$
0<v_{*} \leq v(x) \leq v^{*}<+\infty
$$

and

$$
\inf _{M \backslash \Omega} \bar{S}(x)=\bar{S}_{*}>0 .
$$


5.2.3. Compact support property of bounded solutions of PDEs. Let us recall that a certain PDE satisfies the compact support principle if a solution, in the exterior of a compact set, which is nonnegative and decays at infinity, must have compact support. We are going to analyze some situations where the decay assumption can be relaxed. This has applications to the Yamabe problem.

Theorem 5.15. Let $(M,\langle\rangle$,$) be a geodesically and stochastically complete Cartan-$ Hadamard manifold. Let $u>0$ be a bounded solution of

$$
\Delta u \geq \lambda(u) \quad \text { on } M \backslash \Omega
$$

for a domain $\Omega \subset \subset M$ and for a nondecreasing function $\lambda:[0,+\infty) \rightarrow[0,+\infty)$ satisfying the following conditions:

$$
\text { a) } \lambda(0)=0 ; \quad \text { b) } \lambda(t)>0, \forall t>0 ; \quad \text { c) } \liminf _{t \rightarrow 0+} \frac{\lambda(t)}{t^{\xi}}>0,
$$

for some $0 \leq \xi<1$. Then $u$ has compact support.

Proof. Recall that a Cartan-Hadamard manifold is Feller (see [1] and [20]). By Theorem 4.2, we know that $u(x) \rightarrow 0$, as $x \rightarrow \infty$. The conclusion now follows from the compact support principle, which is valid under the stated assumptions on $M$ and $\lambda$ (see Theorem 1.1 of [22], and Theorem 5.7 above).

The above theorem can be applied to obtain nonexistence results. For instance, combining Theorem 5.15 and Corollary 5.14 we get:

Corollary 5.16. Let $(M,\langle\rangle$,$) be a stochastically complete Cartan-Hadamard m-$ manifold with $m \geq 6$. Then the metric of $M$ cannot be conformally deformed to a new metric, $\overline{\langle,\rangle}=v^{2}\langle$,$\rangle , with v_{*}>0$ and scalar curvature $\bar{S}$ satisfying $\liminf _{x \rightarrow \infty} \bar{S}>0$.

Observe that, for the conclusion of Theorem 5.15 to hold it suffices that $M$ be stochastically complete and Feller, and that the compact support principle holds for solutions of (5.26). Theorem 5.15 can be therefore generalized as follows:

Theorem 5.17. Let $(M,\langle\rangle$,$) be a complete Riemannian manifold with a pole o$ and set $r(x)=d(x, o)$. Assume that

$$
\operatorname{Ric} \geq-K(r(x))
$$

where $K>0$ is an increasing function satisfying

$$
\frac{1}{\sqrt{K}} \notin L^{1}(+\infty)
$$

Assume also that

$$
\mathrm{Sec}_{\mathrm{rad}} \leq G(r(x)),
$$


where $G$ is a smooth even function such that the unique solution $g$ of the Cauchy problem

$$
\left\{\begin{array}{l}
g^{\prime \prime}+G g=0 \\
g(0)=0, \quad g^{\prime}(0)=1
\end{array}\right.
$$

satisfies

$$
\inf \frac{g^{\prime}}{g}(t)>-\infty
$$

If $u \geq 0$ is a bounded solution of

$$
\Delta u \geq \lambda(u) \quad \text { on } M \backslash \Omega,
$$

where $\lambda$ satisfies (5.27), then u has compact support.

In a similar view, using the comparison results established in Theorem 5.1 we obtain the following:

Theorem 5.18. Let $M_{f}$ be a geodesically complete, stochastically complete, and weighted manifold with a pole o, and let $r(x)$ be the Riemannian distance from $o$. Suppose that there exists an integer $n$ and an even function $g: \mathbb{R} \rightarrow[0,+\infty)$ such that $g(0)=0, g^{\prime}(0)=1$, and $g(r)>0$ for $r>0$ such that

$$
\Delta_{f} r(x) \geq(n-1) \frac{g^{\prime}}{g}(r(x)) \quad \text { for } r(x)>>1 .
$$

Suppose moreover

$$
\inf \frac{g^{\prime}}{g}(t)>-\infty
$$

and that either

$$
\frac{1}{g^{n-1}(r)} \in L^{1}(+\infty)
$$

or

$$
\text { (i) } \frac{1}{g^{n-1}(r)} \notin L^{1}(+\infty) \text { and } \quad \text { (ii) } \frac{\int_{r}^{+\infty} g^{n-1}(t) d t}{g^{n-1}(r)} \notin L^{1}(+\infty) \text {. }
$$

If $u>0$ is a bounded solution of

$$
\Delta_{f} u \geq \lambda(u) \quad \text { on } M \backslash \Omega,
$$

where $\lambda$ satisfies (5.27), then u has compact support.

Proof. Indeed, since $g$ satisfies the conditions (5.28) or (5.29), it follows from Theorem 5.1 (iii) that $M_{f}$ is Feller. On the other hand, by Theorem 5.7 the compact support principle holds for solutions of (5.26). Therefore the conclusion follows as in Theorem 5.15. 


\section{Spectral theory of weighted Laplacians}

\subsection{Basic theory}

In this section we collect some results on the spectral properties of the $f$-Laplacian. Generally the proofs may be obtained by adapting those valid for the ordinary Laplacian, and therefore they mostly will be omitted.

The first basic observation is that the $f$-Laplacian is associated to the $f$-Diriclet form

$$
Q_{f}(u)=\int_{M}|\nabla u|^{2} d \mathrm{vol}_{f}
$$

originally defined on $C_{c}^{\infty}(M)$. The form $Q_{f}$ is closable and its closure induces a nonnegative self-adjoint operator on $L^{2}\left(d \operatorname{vol}_{f}\right)$, still denoted $-\Delta_{f}$. The same proof valid for the usual Laplacian can be adapted to show that $-\Delta_{f}$ is essentially selfadjoint on $C_{c}^{\infty}(M)$. It is also useful to note that under the unitary transformation $T(u)=e^{-f / 2} u$ of $L^{2}(d \mathrm{vol})$ onto $L^{2}\left(d \mathrm{vol}_{f}\right)$, the operator $\Delta_{f}$ is unitarily equivalent to the Schrödinger operator

$$
\Delta+\left(\frac{1}{2} \Delta f-\frac{1}{4}|\nabla u|^{2}\right) .
$$

More generally, if $\Omega$ is any open set in $M$, we will denote by $-\Delta_{f}^{\Omega}$ the Friedrichs extension of the operator $-\Delta_{f}$ originally defined on $C_{c}^{\infty}(\Omega)$. Its domain is given by

$$
\operatorname{Dom}\left(-\Delta_{f}^{\Omega}\right)=\left\{u \in H_{0}^{1}\left(\Omega, d \operatorname{vol}_{f}\right):\left(\Delta_{f}\right)_{\operatorname{dist}} u \in L^{2}\left(\Omega, d \operatorname{vol}_{f}\right)\right\} .
$$

The operator $-\Delta_{f}^{\Omega}$ is a positive operator, its spectrum is a subset of $[0,+\infty)$, and the bottom of its spectrum admits the usual variational characterization

$$
\lambda_{1}\left(-\Delta_{f}^{\Omega}\right)=\inf \frac{\int_{\Omega}|\nabla u|^{2} d \operatorname{vol}_{f}}{\int_{\Omega}|u|^{2} d \operatorname{vol}_{f}},
$$

where the infimum is taken over $u \in C_{c}^{\infty}(\Omega)$, or equivalently, in $H_{0}^{1}(\Omega)$.

Also, $\sigma\left(-\Delta_{f}^{\Omega}\right)$ can be decomposed into the disjoint union $\sigma_{d}\left(-\Delta_{f}^{\Omega}\right) \cup \sigma_{\mathrm{ess}}\left(-\Delta_{f}^{\Omega}\right)$, where $\sigma_{d}\left(-\Delta_{f}^{\Omega}\right)$ is the set of isolated eigenvalues of finite multiplicity, called the discrete spectrum, and its complement $\sigma_{\mathrm{ess}}\left(-\Delta_{f}^{\Omega}\right)$, called the essential spectrum, is the set of eigenvalues of infinite multiplicity and of accumulation points of the spectrum.

Adapting the arguments valid for the ordinary Laplacian (or using the above mentioned unitary equivalence with a Schrödinger operator, see [26]) one shows that the following decomposition principle holds:

Theorem 6.1. For every relatively compact domain $\Omega$,

$$
\sigma_{\mathrm{ess}}\left(-\Delta_{f}^{M}\right)=\sigma_{\mathrm{ess}}\left(-\Delta_{f}^{M \backslash \bar{\Omega}}\right) .
$$

In particular,

$$
\inf \sigma_{\mathrm{ess}}\left(-\Delta_{f}^{M}\right)=\sup _{\Omega \subset \subset M} \lambda_{1}\left(-\Delta_{f}^{M \backslash \bar{\Omega}}\right)
$$


Similarly, one may generalize a result of R. Brooks, [7], [8], to obtain the following upper bound for the infimum of the essential spectrum in terms of the weighted volume growth of the manifold (see [26]).

Theorem 6.2. Let $M_{f}$ be a complete weighted manifold.

(a) If $\operatorname{vol}_{f}(M)=+\infty$, then

$$
\limsup _{R \rightarrow+\infty} \frac{\log \operatorname{vol}_{f}\left(B_{R}\right)}{R} \geq \inf \sigma_{\mathrm{ess}}\left(-\Delta_{f}^{M}\right) \geq \lambda_{1}\left(-\Delta_{f}^{M}\right) \geq 0 .
$$

(b) If $\operatorname{vol}_{f}(M)<+\infty$, then

$$
\limsup _{R \rightarrow+\infty} \frac{-\log \left(\operatorname{vol}_{f}(M)-\operatorname{vol}_{f}\left(B_{R}\right)\right)}{R} \geq \inf \sigma_{\mathrm{ess}}\left(-\Delta_{f}^{M}\right) \geq \lambda_{1}\left(-\Delta_{f}^{M}\right) \geq 0 .
$$

The following Barta-type lower estimate for $\lambda_{1}\left(-\Delta_{f}^{\Omega}\right)$ is a weighted version of a result in [3]. Its proof is obtained following exactly the arguments in [3] using a weighted version of the divergence theorem.

Theorem 6.3. Let $M_{f}$ be a weighted manifold and let $\Omega \subset M$ be a domain. Then, for every vector field $X$ on $\Omega$,

$$
\lambda_{1}\left(-\Delta_{f}^{\Omega}\right) \geq \inf _{\Omega}\left\{\operatorname{div}_{f}(X)-|X|^{2}\right\} .
$$

Equality holds if $\Omega$ is a compact domain with smooth boundary.

A classical consequence is given by the next corollary:

Corollary 6.4. Let $M_{f}$ be a weighted manifold and let $\Omega \subset M$ be a domain. Then, for every domain $\Omega$ and for every $0<u \in C^{2}(\Omega)$,

$$
\lambda_{1}\left(-\Delta_{f}^{\Omega}\right) \geq \inf _{\Omega}\left(-\frac{\Delta_{f} u}{u}\right) .
$$

In particular, recalling Theorem 6.1, we deduce:

Corollary 6.5. Let $M_{f}$ be a weighted manifold. Then, for every domain $\Omega \subset \subset M$ and for every $0<u \in C^{2}(M \backslash \Omega)$, there holds

$$
\inf \sigma_{\mathrm{ess}}\left(-\Delta_{f}^{M}\right) \geq \inf _{M \backslash \Omega}\left(-\frac{\Delta_{f} u}{u}\right) .
$$

The following version of the classical Cheng eigenvalue comparison was pointed out in [25].

Theorem 6.6. Assume that the complete weighted manifold $M_{f}$ satisfies

$$
\operatorname{Ric}_{f} \geq-\alpha \text { and }|\nabla f| \leq \beta^{1 / 2}
$$

for some $\alpha, \beta \geq 0$. Then

$$
\lambda_{1}\left(-\Delta_{f}^{B_{R}}\right) \leq \lambda_{1}\left(-\Delta^{\mathbb{B}_{R}^{m+1}}\right),
$$

where $\mathbb{B}_{R}^{m+1}$ is the ball of radius $R>0$ in the $(m+1)$-dimensional spaceform $\mathbb{M}^{m+1}((\alpha+\beta) / m)$ of constant curvature $(\alpha+\beta) / m$. 


\subsection{Essential spectrum and stochastic properties}

The purpose of this section is to find possible connections between the structure of the spectrum of the Laplacian and the stochastic properties of noncompact Riemannian manifold, namely, stochastic (in)completeness and the Feller property. The starting point is the (proof of the) following recent result, [4], which answers in the affirmative a question raised by S. T. Yau.

Theorem 6.7. Let $M$ be a geodesically complete manifold which admits a proper minimal immersion $f: M \rightarrow \mathbb{B}_{R}(0)$ into an open ball $\mathbb{B}_{R}(0) \subset \mathbb{R}^{N}$. Then the essential spectrum is empty, $\sigma_{\mathrm{ess}}\left(-\Delta^{M}\right)=\emptyset$.

Proof. According to the decomposition principle,

$$
\inf \sigma_{\mathrm{ess}}\left(-\Delta^{M}\right)=\sup _{\substack{\Omega_{j} \operatorname{cpt} \\ \Omega_{j} \nearrow M}} \lambda_{1}\left(-\Delta^{M \backslash \Omega_{j}}\right),
$$

where, by the Barta theorem,

$$
\lambda_{1}\left(-\Delta^{M \backslash \Omega_{j}}\right) \geq \sup \inf _{M \backslash \Omega_{j}}\left(-\frac{\Delta v}{v}\right),
$$

the supremum being taken with respect to all smooth (say $C^{2}$ ) functions $v>0$ on $M \backslash \Omega_{j}$. In particular, choosing

$$
\Omega_{j}=\left\{x \in M:|f|^{2} \leq R^{2}-\frac{1}{j}\right\} \subset \subset M
$$

and

$$
v(x)=R^{2}-|f|^{2}>0
$$

gives

$$
\inf \sigma_{\text {ess }}\left(\Delta^{M}\right) \geq \lambda_{1}\left(\Delta^{M \backslash \Omega_{j}}\right) \geq \frac{2 m}{1 / j} \rightarrow+\infty, \quad \text { as } j \rightarrow+\infty \text {. }
$$

Remark 6.8. In the assumptions of Theorem $6.7, M$ is stochastically incomplete. Indeed

$$
u(x)=|f|^{2}
$$

is bounded and satisfies

$$
\Delta u=2 m
$$

thus proving that $u$ violates the weak maximum principle at infinity $(u$ is a "woymp" violating function, in the terminology of [2]). By the characterization of stochastic completeness via the maximum principle, it follows that $M$ is stochastically incomplete, as claimed. Moreover, $0 \leq u(x)<\sup _{M} u=R^{2}<+\infty$ and $\Omega_{\delta}=\left\{x \in M:-\infty<u(x) \leq R^{2}-\delta\right\}$ defines a compact exhaustion of $M$, that is, $u: M \rightarrow\left[0, R^{2}\right)$ is a proper function. We are going to prove that these ingredients suffice to conclude the discreteness of the spectrum, thus establishing an abstract and generalized version of the main Theorem 6.7. 
Definition 6.9. Say that a function $u: M \rightarrow\left(-\infty, u^{*}\right), u^{*}<+\infty$, is a bounded exhaustion function if, for every $\delta>0$, the set

$$
\Omega_{\delta}=\left\{x \in M:-\infty<u(x) \leq u^{*}-\delta\right\}
$$

is compact and $\Omega_{\delta} \nearrow M$ as $\delta \rightarrow 0$. Note that, in case that $M$ is noncompact, necessarily $u^{*}=\sup _{M} u$.

Definition 6.10. Let $M_{f}$ be a weighted manifold. A woymp violating function for the $f$-Laplacian is a $C^{2}$ function $u: M \rightarrow \mathbb{R}$ satisfying $\sup _{M} u=u^{*}<+\infty$ such that, for any sequence $\left\{x_{k}\right\}$ along which $u\left(x_{k}\right) \rightarrow u^{*}$, there holds

$$
\limsup _{k \rightarrow+\infty} \Delta_{f} u\left(x_{k}\right)>0 \text {. }
$$

Theorem 6.11. Let $M_{f}$ be a ( $f$-stochastically incomplete) noncompact weighted manifold. If $M_{f}$ supports a woymp violating exhaustion function then

$$
\sigma_{\mathrm{ess}}\left(-\Delta_{f}^{M}\right)=\emptyset
$$

Proof. Let $u: M \rightarrow\left(-\infty, u^{*}\right), u^{*}=\sup _{M} u<+\infty$, be a woymp violating exhaustion function. Arguing exactly as above, we consider the sets

$$
\Omega_{j}=\left\{x \in M: u(x) \leq u^{*}-1 / j\right\} \nearrow M
$$

and $v(x)=u^{*}-u(x)>0$. Note that, since $u$ is woymp violating,

$$
\inf _{M \backslash \Omega_{j}} \Delta_{f} u=c_{j}>0,
$$

where, by the obvious monotonicity property of the infimum, the sequence $c_{j}$ is increasing. Therefore,

$$
\begin{aligned}
\inf \sigma_{\mathrm{ess}}\left(-\Delta_{f}^{M}\right) & \geq \lambda_{1}\left(-\Delta_{f}^{M \backslash \Omega_{j}}\right) \\
& \geq \inf _{M \backslash \Omega_{j}}\left(-\frac{\Delta_{f} v}{v}\right) \geq j c_{j} \rightarrow+\infty \quad \text { as } j \rightarrow+\infty .
\end{aligned}
$$

At this point, a natural question is to what extent the existence of a woymp violating exhaustion function characterizes the spectrum of the Laplacian of the underlying manifold. Beside proper bounded submanifolds with controlled mean curvature, are there natural examples of manifolds supporting woymp violating exhaustion functions? Which geometric conditions ensure that such functions exist? Some examples will help us to isolate some important aspects.

Example 6.12. Let $M_{g}^{m} \approx \mathbb{R}^{m}$ be a complete, noncompact model manifold endowed with the metric

$$
\langle,\rangle=d r^{2}+g(r)^{2} d \theta^{2}
$$


where the smooth function $g:[0,+\infty) \rightarrow[0,+\infty)$ satisfies

$$
\left\{\begin{aligned}
g(r)>0, & r>0 \\
g^{(2 k)}(0) & =0, \quad k \in \mathbb{N} \\
g^{\prime}(0) & =1 .
\end{aligned}\right.
$$

According to a well-known characterization, $M_{g}^{m}$ is stochastically incomplete if and only if

$$
\int_{0}^{+\infty} \frac{\int_{0}^{t} g^{m-1}}{g^{m-1}(t)}=u^{*}<+\infty
$$

Indeed, the function,

$$
u(x)=\int_{0}^{r(x)} \frac{\int_{0}^{t} g^{m-1}}{g^{m-1}(t)} d t: M_{g}^{m} \rightarrow\left[0, u^{*}\right),
$$

satisfies

$$
\Delta u=1,
$$

and it is bounded if and only (6.2) holds, in which case $u$ is in fact a woymp violating exhaustion function, and $\sigma_{\text {ess }}\left(-\Delta^{M_{g}^{m}}\right)=\emptyset$.

Thus a model is stochastically incomplete if and only if it admits a woymp violating exhaustion function. In particular, for a stochastically incomplete model, the condition that $\sigma_{\text {ess }}\left(-\Delta^{M_{g}^{m}}\right)=\emptyset$ is equivalent with the existence of a woymp violating exhaustion.

Example 6.13. In general it is not true that the stochastic incompleteness of a manifold is equivalent to the discreteness of the spectrum of its Laplacian. Indeed, condition the $\sigma_{\text {ess }}\left(-\Delta^{M}\right)=\emptyset$ is invariant under bilipschitz diffeomorphisms whereas, according to a result by T. Lyons, [15], stochastic (in)completeness is not. More concretely, consider the Riemannian product $N=M_{g}^{m} \times \mathbb{R}$ where $M_{g}^{m}$ is stochastically incomplete model. Then, $N$ is stochastically incomplete but the essential spectrum of the Laplacian $\Delta_{N}=\Delta_{M}+d^{2} / d t^{2}$ is nonempty. Note that a natural voymp violating function on $N$ is given by $v(x, t)=u(x)$ where $u(x)$ is defined in the previous example. Clearly, $v: N \rightarrow\left[0, u^{*}\right)$ is not an exhaustion function. This shows that the assumptions of Theorem 6.11 are necessary.

One may also investigate whether the condition that the bottom of the spectrum of exterior balls grows at a specified rate, namely

$$
\lambda_{1}\left(-\Delta^{M \backslash \bar{B}_{R}}\right) \geq f(R),
$$

where $f(R)$ is a monotone nondecreasing function and diverges as $R \rightarrow+\infty$, forces stochastic incompleteness. However, without additional global assumptions even this implication fails. To see this, we begin by observing that, by standard arguments, if $(M,\langle\rangle$,$) has a pole o$ and $|\Delta r| \geq c>0$ on $M \backslash \bar{\Omega}$, then $\lambda_{1}\left(-\Delta^{M \backslash \bar{\Omega}}\right) \geq c^{2} / 4$. 
Indeed, by continuity $\Delta r$ has constant sign in every connected component of $M \backslash \bar{\Omega}$. For every $v \in C_{c}^{\infty}(M \backslash \bar{\Omega})$ with support in one of such connected components we have

$$
\int v^{2} \leq \frac{1}{c} \int|\Delta r| v^{2}= \pm \frac{2}{c} \int v\langle\nabla v, \nabla r\rangle \leq \frac{2}{c}\left(\int v^{2}\right)^{1 / 2}\left(\int|\nabla v|^{2}\right)^{1 / 2},
$$

and

$$
\frac{c^{2}}{4} \int v^{2} \leq \int|\nabla v|^{2}
$$

Note that it follows from the decomposition principle, that if $|\Delta r| \rightarrow+\infty$ as $x \rightarrow \infty$, then $\sigma_{\text {ess }}\left(-\Delta^{M}\right)=\emptyset$.

Now, given a function $f$ as above, assume that $g$ satisfies

$$
g(r)=\exp \left(-2 \int_{1}^{r} \sqrt{f(t)} d t\right)
$$

for sufficiently large $r$. Then the model $M_{g}^{m}$ has the property that

$$
\Delta r \leq-2 \sqrt{f(R)} \text { in the complement of } B_{R},
$$

and (6.3) follows. On the other hand, since $g(r)$ is decreasing, $\operatorname{vol}\left(B_{R}\right)$ grows at most linearly, and $M_{g}^{m}$ is necessarily stochastically complete.

We note in passing that this shows that the assumption that $M$ be a CartanHadamard manifold plays a fundamental role in Problem 10 of Grigor'yan's survey [12].

Example 6.14. In general, a stochastically incomplete manifold with discrete spectrum may support woymp violating functions both exhaustion and not. Indeed, take $N=M \times M$ where $M$ supports a positive woymp violating exhaustion function $u: M \rightarrow\left[0, u^{*}\right.$ ) (for instance, $M$ is one of the bounded minimal surfaces of $\mathbb{R}^{3}$ of Martín-Morales). Then $\sigma_{\text {ess }}\left(-\Delta^{M}\right)=\emptyset$. Moreover, $v(x, y)=u(x)+u(y)$ is woymp violating and exhaustion whereas $w(x, y)=u(x)$ is woymp violating but not exhaustion. As a consequence, for a stochastically incomplete manifold $M$, the condition $\sigma_{\text {ess }}\left(-\Delta^{M}\right)=\emptyset$ could imply at most that there exists one woymp violating exhaustion function.

In a similar manner, since both $\sigma_{\text {ess }}$ and the Feller property are only sensitive to the properties of the manifold off a compact set, it is also natural to investigate to what extent they are related. However, we are going to see that, without further assumptions, there is no link between the Feller property and the discreteness of the spectrum.

Example 6.15. For simplicity, we restrict ourselves to the case of the ordinary Laplacian $\Delta$, even though much of the ensuing discussion could be generalized to the $f$-Laplacian. 
As noted in the proof of Theorem 5.18, the model manifold $M_{g}^{m}$ is Feller if and only if either

$$
\frac{1}{g^{m-1}(r)} \in L^{1}(+\infty)
$$

or

$$
\text { (i) } \frac{1}{g^{m-1}(r)} \notin L^{1}(+\infty) \text { and (ii) } \frac{\int_{r}^{+\infty} g^{m-1}(t) d t}{g^{m-1}(r)} \notin L^{1}(+\infty) \text {. }
$$

Recalling that $\Delta r=(m-1) g^{\prime} / g$, we deduce from the discussion in Example 6.13 that, if $g(r)=e^{-r^{\alpha}}$ for $r>>1$, then $M_{g}^{m}$ has discrete spectrum for every $\alpha>1$, and it is Feller for $\alpha \leq 2$ and non-Feller for $\alpha>2$. Note that all these manifolds have finite volume, and so are automatically stochastically complete, showing that even in the case of models there is no equivalence between discreteness of the spectrum, and stochastic incompleteness.

It follows that if $M$ is the connected sum of $\mathbb{R}^{m}$ with a non-Feller model $M_{g}^{m}$, then $M$ is non-Feller. Since the essential spectrum of $\mathbb{R}^{m}$ is the entire interval $[0,+\infty)$ an easy argument based on characteristic sequences supported in the end of $M$ isometric to an exterior domain of $\mathbb{R}^{m}$ shows that $\sigma_{\mathrm{ess}}\left(-\Delta^{M}\right)=[0,+\infty)$, and $M$ is therefore a non-Feller manifold with non-empty essential spectrum. Of course, $\mathbb{R}^{m}$ itself is a trivial example of a Feller manifold with non-empty essential spectrum.

Example 6.16. As seen in Example 6.12, a stochastically incomplete model has discrete spectrum. Since such model has necessarily infinite volume, it is Feller by the characterization of the previous example. Small modifications of the arguments described above show that even for non-Feller, stochastically incomplete manifolds there is in general no connection with the discreteness of the spectrum.

Recall that one may extend the definition of stochastic (in)completeness to an end of a manifold, by requiring that the double of the end be stochastically complete (incomplete). Then it follows easily from the weak maximum principle that a manifold is stochastically incomplete if and only if so is at least one of its ends.

The connected sum $M=M_{g_{1}}^{m} \# M_{g_{2}}^{m}$ of a stochastically incomplete model $M_{g_{1}}^{m}$ with a non-Feller model $M_{g_{2}}^{m}$ with discrete spectrum, as described in Example 6.15, provides an example of a stochastically incomplete, non-Feller manifold with empty essential spectrum, $\sigma_{\text {ess }}\left(-\Delta^{M}\right)=\emptyset$.

On the other hand, the connected sum $M=M_{g_{1}}^{m} \# M_{g_{2}}^{m} \# \mathbb{R}^{m}$ is stochastically incomplete, non-Feller, and $\sigma_{\text {ess }}\left(-\Delta^{M}\right)=[0,+\infty)$.

Note that all these examples have more than one end, and the case of manifolds with only one end remains open.

\subsection{Spectrum and semilinear PDEs}

In this section we use very easy spectral considerations to deduce information on nonnegative solutions of the differential inequality

$$
\Delta_{f} u \leq a u-b u^{\sigma},
$$


in the exterior of a compact set for some constants $a \geq 0, b>0$ and $\sigma>1$. By the strong minimum principle, $u>0$ unless it is identically zero in each connected component of every point where it vanishes. If $u$ satisfied the inequality on all of $M_{f}$, and the weighted manifold was stochastically complete with respect to the $f$-Laplacian, then a direct application of the weak maximum principle at infinity would imply that either $\inf _{M} u=0$ or

$$
\inf _{M} u \leq\left(\frac{a}{b}\right)^{1 /(\sigma-1)}
$$

For instance, when applied to the scalar curvature $S(x)$ of a complete, shrinking Ricci soliton, this procedure gives the estimate

$$
0 \leq \inf _{M} S \leq m \lambda
$$

where $\lambda$ denotes the soliton constant and $m=\operatorname{dim} M$. Indeed, it is well known that $S$ satisfies

$$
\frac{1}{2} \Delta_{f} S=\lambda S-|\mathrm{Ric}|^{2} \leq \lambda S-\frac{S^{2}}{m} .
$$

In the spirit of the previous sections, we are going to extend these considerations outside a compact set. This time, however, we use spectral assumptions instead of stochastic properties.

Proposition 6.17. Let $u>0$ be a solution of (6.5) in a neighborhood at infinity, for some constants $a \in \mathbb{R}, b>0$ and $\sigma>1$. Then, for every domain $\Omega \subset \subset M$,

$$
\inf _{M \backslash \Omega} u \leq\left(\frac{a+\inf \sigma_{\mathrm{ess}}\left(-\Delta_{f}^{M}\right)}{b}\right)^{1 /(\sigma-1)} .
$$

Remark 6.18. In particular, if inf $\sigma_{\text {ess }}\left(-\Delta_{f}^{M}\right)=0$, we recover the conclusion above outside every large compact set. According to Brooks' estimates [7], this happens under suitable volume growth assumptions. If $\inf _{M \backslash \Omega} u=(a / b)^{1 /(\sigma-1)}$, then $\Delta_{f} u \leq 0$ on $M \backslash \Omega$ and therefore by the comparison principle $u>(b / a)^{1 /(\sigma-1)}$ in the interior of $M \backslash \Omega$. We then conclude that $\inf _{M \backslash \Omega} u=(a / b)^{1 /(\sigma-1)}$ for every $\Omega^{\prime} \supseteq \Omega$, so that the infimum is attained at infinity.

Conversely, if $\inf _{M \backslash \Omega} u>(a / b)^{1(\sigma-1)}$, we deduce a gap in the essential spectrum and, therefore, a volume growth estimate. In particular, this is the case when $a<0$. From another point of view, when $a<0$ or when $b<0$ the estimate may be interpreted as a non-existence result.

Proof. This is a trivial application of Corollary 6.5. Indeed

$$
\inf \sigma_{\mathrm{ess}}\left(-\Delta_{f}^{M}\right) \geq \inf _{M \backslash \Omega}\left(-\frac{\Delta_{f} u}{u}\right) \geq-a+b \inf _{M \backslash \Omega} u^{\sigma-1}
$$


Returning to the scalar curvature $S(x)$ of a gradient shrinker $M_{f}$ with soliton constant $\lambda>0$, recall that $S \geq 0$, the equality holding at some point if and only if

$$
M_{f}=\mathbb{R}_{f}^{m} \quad \text { and } \quad f(x)=A|x|^{2}+\langle B, x\rangle+c .
$$

Assume $S>0$, for otherwise there is nothing to prove. Then, for every $R>0$, we obtain the estimates

$$
\inf _{M \backslash B_{R}} \frac{|\mathrm{Ric}|^{2}}{S}-\lambda \leq \inf \sigma_{\mathrm{ess}}\left(-\Delta_{f}^{M}\right)
$$

and

$$
\inf _{M \backslash B_{R}} S-m \lambda \leq m \inf \sigma_{\mathrm{ess}}\left(-\Delta_{f}^{M}\right)
$$

In the case of an expanding Ricci soliton, we have $m \lambda \leq \inf _{M} S \leq 0$. Assume that $\inf _{M \backslash B_{R_{o}}} S(x) \geq 0$ for some $R_{o} \geq 0$, so that we must have either $\inf _{M} S=$ $\inf _{B_{R_{o}}} S<0$ or $\inf _{M} S=0$ and the infimum is not attained. Exactly as before, the estimates (6.8) and (6.9) hold for every $R \geq R_{o}$ and from the latter we deduce in particular that inf $\sigma_{\text {ess }}\left(-\Delta_{f}^{M}\right)>0$ provided $S>0$ outside a compact set.

To conclude this section, we remark that Proposition 6.17 follows essentially from a suitable application of Barta's theorem. Further use of this result, but in a slightly different direction, yields a different kind of information on the solutions at infinity of (6.5).

Proposition 6.19. Let $M_{f}$ be a complete, m-dimensional weighted manifold satisfying

$$
\operatorname{Ric}_{f} \geq-\mu \quad \text { and } \quad|\nabla f| \leq \beta^{1 / 2}
$$

for some constants $\mu>0, \beta \geq 0$. Then, there exists a constant $c=c(m, \mu, \beta)>0$ such that the following holds. If $u$ is a solution of (6.5) outside a compact set $K$, for some constants $a \geq 0, b>0$ and $\sigma>1$, then, for every $B_{R}(x) \in M \backslash K$,

$$
\left(\frac{a}{b}+\frac{c}{b} \frac{1+R^{2}}{R^{2}}\right)^{1 /(\sigma-1)} \geq \inf _{B_{R}(x)} u .
$$

In particular, for any fixed $R>0$,

$$
\limsup _{x \rightarrow \infty}\left\{\inf _{B_{R}(x)} u\right\}<+\infty .
$$

Proof. Obviously, the only interesting case is

$$
\inf _{B_{R}(x)} u>0 .
$$

Then,

$$
\lambda_{1}\left(-\Delta_{f}^{B_{R}(x)}\right) \geq \inf _{B_{R}(x)}\left(-\frac{\Delta_{f} u}{u}\right) \geq-a+b \inf _{B_{R}(x)} u^{\sigma-1}
$$


On the other hand, by Theorem 6.6,

$$
\lambda_{1}\left(-\Delta_{f}^{B_{R}(x)}\right) \leq \lambda_{1}\left(-\Delta^{\mathbb{B}_{R}^{m+1}}\right) \leq c_{1}(\mu, \beta, m)\left(1+\frac{1}{R^{2}}\right)
$$

with $\mathbb{B}_{R}^{m+1} \subset \mathbb{H}^{m+1}((\beta+\mu) / m)$. Combining these two inequalities completes the proof.

The above result does not apply as stated to expanding Ricci solitons, since in this case $|\nabla f|$ cannot be bounded unless the soliton is trivial (see [18]). However, by Zhang's estimates, $|\nabla f|$ grows at most linearly in the distance from a reference point (and indeed, it was very recently shown by O. Munteanu and J.P. Wang that its growth is in fact essentially linear).

Assuming that $|\nabla f| \leq C_{o} r(x)$ in the above argument, it follows that for every fixed $R$ and every $x$ such that $r(x) \geq 2 R$

$$
\lambda_{1}\left(-\Delta_{f}^{B_{R}(x)}\right) \leq c_{1}\left(\mu+C^{2}(R+r(x))^{2}\right)\left(1+\frac{1}{R^{2}}\right)
$$

for some constant $C$ depending only on $m$, and we conclude that if $u$ is as in the statement of Proposition 6.19 then there exists a constant $c_{2}$ depending on $m, \mu$, $\sigma, C_{o}$ and $R$ such that

$$
\frac{\inf _{B_{R}} u}{r(x)^{2}} \leq c_{2}
$$

This in particular holds for expanding Ricci solitons and can be compared with Zhang's estimate

$$
\frac{|S(x)|}{r(x)^{2}} \leq d(m, \lambda) .
$$

Note that the latter follows applying the estimates of the potential function to the basic equation (5.23), and is therefore specific to the much more rigid geometry imposed by the soliton structure.

\section{References}

[1] Azencott, R.: Behavior of diffusion semi-groups at infinity. Bull. Soc. Math. France 102 (1974), 193-240.

[2] B̈̈r, C. And Bessa, G. P.: Stochastic completeness and volume growth. Proc. Amer. Math. Soc. 138 (2010), no. 7, 2629-2640.

[3] Bessa, G. P. And Montenegro, J. F.: An extension of Barta's theorem and geometric applications. Ann. Global Anal. Geom. 31 (2007), no. 4, 345-362.

[4] Bessa, G. P., Jorge, L. P. And Montenegro, J. F.: The spectrum of the MartínMorales-Nadirashvili minimal surfaces is discrete. J. Geom. Anal. 20 (2010), no. 1, 63-71.

[5] BorbéLY, A.: Immersion of manifolds with unbounded image and a modified maximum principle of Yau. Bull. Aust. Math. Soc. 78 (2008), no. 2, 285-291. 
[6] Borbély, A.: On minimal surfaces satisfying the Omori-Yau principle. Bull. Aust. Math. Soc. 84 (2011), no. 1, 33-39.

[7] Brooks, R.: A relation between growth and the spectrum of the Laplacian. Math. Z. 178 (1981), no. 4, 501-508.

[8] Brooks, R.: On the spectrum of noncompact manifolds with finite volume. Math. Z. 187 (1984), no. 3, 425-432.

[9] CaO, H. D. and Zhou, D.: On complete gradient shrinking Ricci solitons. J. Differential Geom. 85 (2010), no. 2, 175-185.

[10] Fernández-López, M. And García-Río, E.: Maximum principles and gradient Ricci solitons. J. Differential Equations 251 (2011), no. 1, 73-81.

[11] Grigor'yan, A.: Stochastically complete manifolds and summable harmonic functions. Izv. Akad. Nauk SSSR Ser. Mat. 52 (1988), no. 5, 1102-1108. Translation in Math. USSR-Izv. 33 (1989), no. 2, 425-432.

[12] Grigor'yan, A.: Analytic and geometric background of recurrence and nonexplosion of the Brownian motion on Riemannian manifolds. Bull. Amer. Math. Soc. (N.S.) 36 (1999), no. 2, 135-249.

[13] Harmer, M.: Discreteness of the spectrum of the Laplacian and stochastic incompleteness. J. Geom. Anal. 19 (2009), no. 2, 358-372.

[14] Hsu, P.: Heat semigroup on a complete Riemannian manifold. Ann. Probab. 17 (1989), no. 3, 1248-1254.

[15] Lyons, T: Instability of the conservative property under quasi-isometries. J. Differential Geom. 34 (1991), no. 2, 483-489.

[16] Pigola, S., Rigoli, M. And Setti, A. G.: Volume growth, "a priori" estimates, and geometric applications. Geom. Funct. Anal. 13 (2003), no. 6, 1302-1328.

[17] Pigola, S., Rigoli, M. And Setti, A. G.: Maximum principles on Riemannian manifolds and applications. Mem. Amer. Math. Soc. 174 (2005), no. 822, x+99.

[18] Pigola, S., Rimoldi, M. and Setti, A. G.: Remarks on non-compact gradient Ricci solitons. Math. Z. 268 (2011), no. 3-4, 777-790.

[19] Pigola, S., Rigoli, M., Rimoldi, M. and Setti, A. G.: Ricci almost solitons. Ann. Sc. Norm. Super. Pisa Cl. Sci. (5) 10 (2011), no. 4, 757-799.

[20] Pigola, S. And Setti, A. G.: The Feller property on Riemannian manifolds. J. Funct. Anal. 262 (2012), no. 5, 2481-2515.

[21] Pucci, P., Serrin, J. Zou, H.: A strong maximum principle and a compact support principle for singular elliptic inequalities. J. Math. Pures Appl. 78 (1999), no. 8, 769-789.

[22] Pucci, P., Rigoli, M. And Serrin, J.: Qualitative properties for solutions of singular elliptic inequalities on complete manifolds. J. Differential Equations 324 (2007), no. 2, 507-543.

[23] Qian, Z.: On conservation of probability and the Feller property. Ann. Probab. 24 (1996), no. 1, 280-292.

[24] Ratto, A., Rigoli, M. And Veron, L.: Scalar curvature and conformal deformations of noncompact Riemannian manifolds. Math. Z. 225 (1997), no. 3, 395-426.

[25] Setti, A. G.: Eigenvalue estimates for the weighted Laplacian on a Riemannian manifold. Rend. Sem. Mat. Univ. Padova 100 (1998), 27-55. 
[26] Volpi, S.: Spectral properties of Schr̈odinger operators. [Italian] Master Thesis. Università dell'Insubria, Como, 2008.

[27] Wei, G. And Wylie, W.: Comparison geometry for the Bakry-Emery Ricci tensor. J. Differential Geom. 83 (2009), no. 2, 377-405.

[28] Zhang, Z. H.: On the completeness of gradient Ricci solitons. Proc. Amer. Math. Soc. 137 (2009), no. 8, 2755-2759.

Received July 6, 2011.

G. Pacelli Bessa: Departamento de Matemática, Universidade Federal do CearáUFC, 60455-760 Fortaleza, CE, Brazil.

E-mail: bessa@mat.ufc.br

Stefano Pigola: Sezione di Matematica-DiSAT, Università dell'Insubria-Como, via Valleggio 11, 22100 Como, Italy.

E-mail: stefano.pigola@uninsubria.it

Alberto Setti: Sezione di Matematica-DiSAT, Università dell'Insubria-Como, via Valleggio 11, 22100 Como, Italy.

E-mail: alberto.setti@uninsubria.it 